\title{
Integrity of plasma cell-free DNA as a prognostic factor for vaccine therapy in patients with endometrial cancer
}

\author{
KAYOKO WAKI $^{1}$, KANAKO YOKOMIZO ${ }^{1}$, KOUICHIRO KAWANO ${ }^{2}$, \\ NAOTAKE TSUDA $^{2}$, NOBUKAZU KOMATSU ${ }^{3}$ and AKIRA YAMADA ${ }^{1}$ \\ ${ }^{1}$ Cancer Vaccine Development Division, Research Center for Innovative Cancer Therapy, \\ Kurume University; ${ }^{2}$ Departments of Obstetrics and Gynecology and ${ }^{3}$ Immunology, \\ Kurume University School of Medicine, Kurume, Fukuoka 830-0011, Japan
}

Received June 2, 2020; Accepted November 18, 2020

DOI: $10.3892 /$ mco.2020.2191

\begin{abstract}
Endometrial cancer is the most prevalent gynecological cancer in developed countries. Although the prognosis of endometrial cancer is better than that of other gynecological cancers, the prognosis of advanced endometrial cancer is still poor and thus new therapeutic modalities, such as immune therapies, are urgently required. For the further development of new modalities, exploration of new biomarkers is important. The present study investigated the circulating cell-free DNA (cfDNA) integrity as a ratio of the necrotic tumor cell-derived long cfDNA fragments to the total dead cell-derived short cfDNA fragments from genomic Alu elements in patients with advanced endometrial cancer during peptide vaccination treatment. The results demonstrated that: i) The plasma cfDNA integrity was decreased during the first cycle of vaccination in patients with endometrial cancer treated with the personalized peptide vaccination, and ii) the post-vaccination cfDNA integrity levels were correlated with good prognosis. Some of these findings have been confirmed in other cancers, and thus cfDNA integrity might be an important marker for future cancer vaccine therapies in general, and might also be applicable for other immune therapies.
\end{abstract}

\section{Introduction}

Endometrial cancer is a malignancy of the corpus uteri and its morbidity is increasing in Japan (1). Currently it is the most prevalent gynecological cancer in Japan as well as in other developed countries. Globally, nearly 382,000 individuals

Correspondence to: Professor Akira Yamada, Cancer Vaccine Development Division, Research Center for Innovative Cancer Therapy, Kurume University, 67 Asahi-machi, Kurume, Fukuoka 830-0011, Japan

E-mail: akiymd@med.kurume-u.ac.jp

Key words: cell-free DNA integrity, endometrial cancer, peptide vaccine, prognosis, immunotherapy, vaccine therapy, biomarker are newly diagnosed with endometrial cancer each year, and $\sim 90,000$ die from it (2). Most patients with endometrial cancer are cured by surgery alone or with adjuvant chemotherapy, and thus the prognosis of endometrial cancer is better than the prognosis of other gynecological cancers (3). However, because the prognosis of advanced endometrial cancer is still poor (3), new therapeutic modalities are urgently needed.

We have developed a peptide vaccine that is personalized according to each patient's HLA-A locus type and pre-vaccination immunity to a vaccine peptide panel (4-6). The peptide panel consists of 31 different cytotoxic T-lymphocyte (CTL)-epitope peptides, and a maximum of 4 peptides are selected and used as vaccines with Montanide ISA51VG adjuvant. Clinical trials of the vaccines, named the personalized peptide vaccines, were conducted in patients with various cancers, including gynecological cancers, and the results showed that the vaccines were both feasible and safe (4-8). For further development of the personalized peptide vaccines, the identification of new biomarkers will be important. A recent trend in the identification of new biomarkers is 'liquid biopsy' using cell-free plasma/serum specimens and circulating tumor cells. Therefore, we focused on circulating cell-free DNA (cfDNA) in the plasma and investigated the cfDNA integrity of patients with advanced endometrial cancer during treatment with the personalized peptide vaccination.

\section{Patients and methods}

Patients and plasma samples. Frozen plasma samples from 32 patients with advanced endometrial cancer who were enrolled in clinical trials of the personalized peptide vaccination during the period from December 2008 to March 2018 were used in this study. Clinical stages of the patients were as follows: Stage III $(n=2)$, stage IV $(n=3)$ and recurrent $(n=27)$. Histology of the patients was as follows: 19 endometrioid carcinoma (7 G1, 7 G2, 5 G3), 8 serous carcinoma, 1 clear cell carcinoma, 1 adenosquamous cell carcinoma, and 1 neuroendocrine carcinoma. Histology of the remaining two patients was unknown. The clinical protocols of the personalized peptide vaccination have been reported previously $(7,8)$. The clinical study was approved by the Kurume University 
A

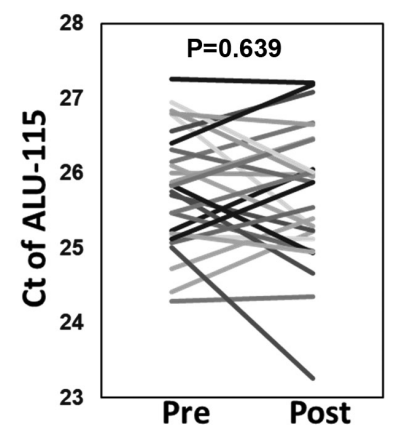

B

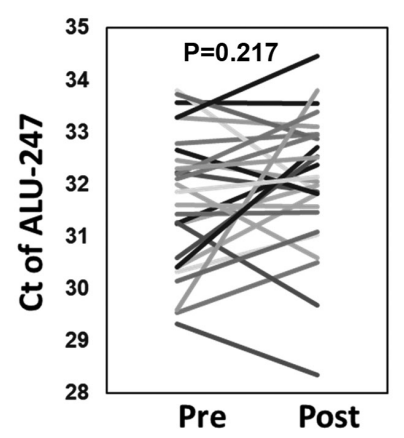

C

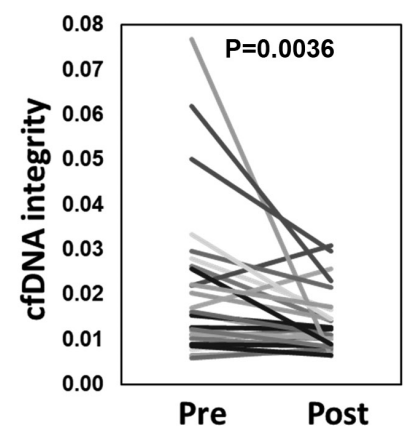

Figure 1. Cycle threshold $(\mathrm{Ct})$ values of PCR fragments of Alu element and cfDNA integrity of plasma samples obtained before (Pre) and after (Post) the first cycle of vaccination. (A) Ct values of total cfDNA (Alu-115), (B) tumor cell-derived cfDNA (Alu-247), (C) the cfDNA integrity (Alu-247/115). ns, not significant; cfDNA, circulating cell-free DNA.

Ethics Committee and registered with the UMIN Clinical Trial Registry under trial numbers UMIN1482, 10068, 11230, and 14855. Written informed consent was obtained from all participants included in the study. The plasma samples used were obtained before and after the first vaccination cycle, which consisted of weekly injection for 6 or 8 weeks.

cfDNA integrity. The analytical method of the cfDNA integrity was described elsewhere $(9,10)$. After thawing the plasma samples, insoluble materials were removed by centrifugation at $16,000 \mathrm{x} \mathrm{g}$ for $5 \mathrm{~min}$ at $4^{\circ} \mathrm{C}$. The supernatants were diluted 1:40 with distilled water, and $1.5 \mu \mathrm{l}$ of each sample was subjected to subsequent polymerase chain reaction (PCR) in a total volume of $15 \mu \mathrm{l}$. Short (115-bp) and long (247-bp) Alu fragments were amplified and quantitatively analyzed using real-time PCR (StepOne plus; Thermo Fisher Scientific, Inc.) with THUNDERBIRD SYBR qPCR mix (Toyobo). The PCR primer pairs were as follows: 5'-CCTGAGGTCAGGAGTTCG AG-3' (forward) and 5'-CCTGAGGTCAGGAGTTCGAG-3' (reverse) for Alu-115; 5'-GTGGCTCACGCCTGTAATC-3' (forward) and 5'-CAGGCTGGAGTGCAGTGG-3' (reverse) for Alu-247. The PCR protocol consisted of an initial denaturation at $95^{\circ} \mathrm{C}$ for $10 \mathrm{~min}$, followed by 40 cycles of amplification at $95^{\circ} \mathrm{C}$ for $30 \mathrm{sec}, 64^{\circ} \mathrm{C}$ for $30 \mathrm{sec}$, and $72^{\circ} \mathrm{C}$ for $30 \mathrm{sec}$. An arbitrary cutoff value of delta $\mathrm{Rn}=0.65$ was used to obtain cycle threshold $(\mathrm{Ct})$ values (11). Short 115-bp and long 247-bp PCR fragments of Alu reflect total cfDNA and necrotic cell (mainly tumor cell)-derived cfDNA, respectively. The cfDNA integrity was calculated according to the formula: cfDNA integrity $=2^{\text {(Ct value of Alu-115-Ct value of Alu-247) }}$.

Measurement of peptide-reactive IgG and CTLs. Quantitation of vaccinated peptide-reactive $\operatorname{IgG}$ in the plasma and CTLs was described previously (7). The IgG responses were measured by the LUMINEX beads method and the CTL responses were measured by an ELISPOT assay of interferon-gamma-secreting cells. If the IgG levels or spot number were increased to $\geq 2$-fold the pre-vaccination level, the response was considered augmented.

Statistical analysis. The cfDNA and cfDNA-integrity levels of the pre- and post-vaccination were compared by Wilcoxon's signed rank test. The relationship between the cfDNA integrity and pathogenetic types were analyzed by Wilcoxon's rank sum test. The survival curves were plotted by the Kaplan-Meier method. We compared the high and low cfDNA-integrity groups at before and after the first vaccination cycle by using a Cox's proportional hazard model. The contribution of other factors, including cfDNA integrity as a continuous variable, to the overall survival (OS) was also analyzed by using a Cox's proportional hazard model. The statistical analyses were performed using JMP Pro version 14 software (SAS, Inc.).

\section{Results}

Alteration of the circulating cfDNA integrity during the peptide vaccination. To analyze the cfDNA integrity, genomic DNA fragments of the Alu element, which is the most abundant repetitive element in the human genome, in the plasma were quantified by real-time PCR. Fig. 1 shows the $\mathrm{Ct}$ values of short 115-bp (Alu-115) and long 247-bp (Alu-247) PCR fragments of the Alu element and cfDNA integrity of plasma samples obtained at before and after the first vaccination cycle. The cfDNA-integrity values after one cycle of vaccination were significantly decreased $(\mathrm{P}=0.0036)$. In contrast, such alteration was not observed in the $\mathrm{Ct}$ values of Alu-115 and 247 . We therefore analyzed the relative contributions of Alu-115 and 247 to the alteration of cfDNA integrity. As shown in Fig. 2, the change in Alu-247, but not Alu-115, was significantly corelated with the change in cfDNA integrity ( $\mathrm{r}=-0.741, \mathrm{P}<0.0001)$.

Relationship between the circulating cfDNA integrity and pathogenetic types. Next, we analyzed the relationship between the cfDNA integrity and pathogenetic types. Pathogenetic classification of the patients were as follows: Type $\mathrm{I}(\mathrm{n}=14)$ consisted of 14 endometrioid carcinoma ( 7 grade 1 and 7 grade 2); type II $(n=14)$ consisted of 5 endometrioid carcinoma grade 3, 8 serous carcinoma, and 1 clear cell carcinoma. As shown in Fig. 3, there were no significant correlation between the pathogenetic types and either the pre-vaccination values, post-one cycle vaccination values, or changes in cfDNA integrity.

Relationship between the circulating cfDNA integrity and prognosis. To examine the relationship between the circulating cfDNA integrity and prognosis, the patients were divided 
A

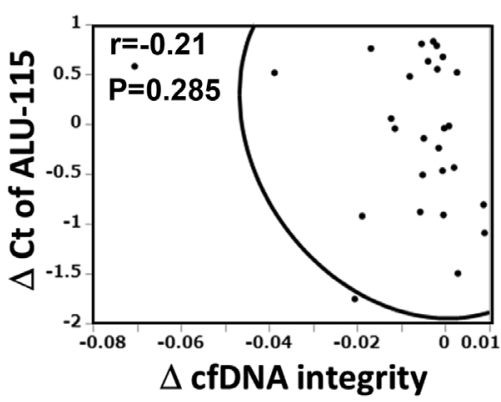

B

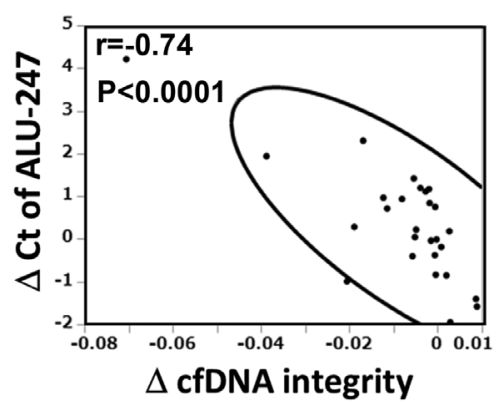

Figure 2. Contribution of the changes in Alu-115 and 247 to the change of cfDNA integrity during vaccination. (A) Relationship between Alu-115 and cfDNA integrity. (B) Relationship between Alu-247 and cfDNA integrity. cfDNA, circulating cell-free DNA.

A

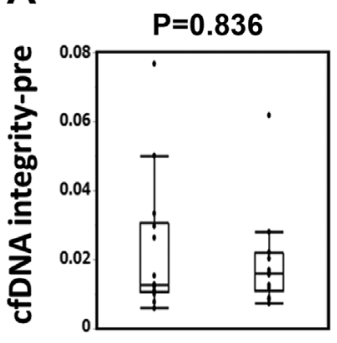

Type I Type II
B

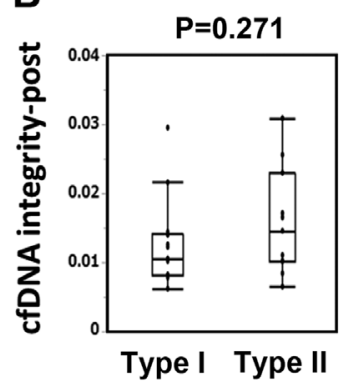

C

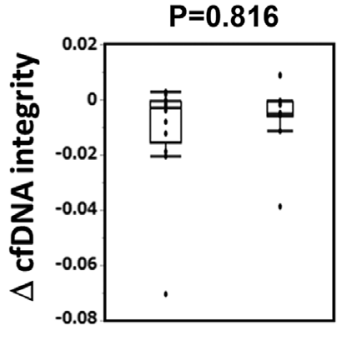

Type I Type II

Pathogenetic classification

Figure 3. Relationship between the cfDNA integrity and pathogenetic types. Plasma samples were obtained before (Pre) and after (Post) the first cycle of vaccination and cfDNA integrity was measured. (A) Pre-vaccination cfDNA integrity. (B) Post-vaccination cfDNA integrity. (C) The difference in cfDNA integrity between pre- and post-vaccination (D cfDNA integrity). cfDNA, circulating cell-free DNA.

into high and low cfDNA-integrity groups and their OS was analyzed by Kaplan-Meier plot. 'High' and 'low' were respectively defined as the upper and lower median values of cfDNA. As shown in Fig. 4, there were no significant correlations between OS and either the pre-vaccination values, post-one cycle vaccination values, or changes in cfDNA integrity. The median survival time (MST) of the post-vaccination high cfDNA-integrity group was longer than that of the low cfDNA-integrity group (40 and 14.3 months, respectively), although the difference was not statistically significant. Thus, we further analyzed the contribution of various factors, including cfDNA integrity as continuous variables, to the OS by Cox's proportional hazard analysis (Table I). Among the various factors, only post-vaccination cfDNA integrity was significantly correlated with OS $(\mathrm{P}=0.038)$. Vaccine-induced IgG or CTL responses were not significantly correlated with OS.

\section{Discussion}

In previous studies, we reported several biomarkers for peptide vaccine therapy against the advanced stage of various cancers (12-18). The vaccines consisted of CTL-epitope peptides, and therefore vaccine-induced CTL responses were the primary mechanism underlying the therapeutic effect of the vaccination, and early induction of the CTL responses to the vaccine peptides was correlated with good prognosis (12-17). Some CTL epitope peptides can induce an
IgG response in the presence of helper T cells. Our peptide vaccines are personalized by each patient's HLA-A locus type and pre-vaccination immunity to the peptide panel detected by $\operatorname{IgG}$ (4-6). Therefore, early induction or augmentation of $\mathrm{IgG}$ responses to the vaccine peptides is also correlated with good prognosis in patients treated with the personalized peptide vaccination (12-17). In contrast, inflammation-related factors such as C-reactive protein (CRP), interleukin (IL)-6, and serum amyloid A (SAA) were correlated with poor prognosis of the vaccine-treated patients (12-17). High mobility group box-1 (HMGB1), a damage-associated molecular pattern, is released from both the dead tumor cells and activated macrophages. Correlation between the plasma levels of HMGB1 and tumor progression has been reported in various cancers (18). The plasma levels of HMGB1 have also been correlated with poor prognosis in patients treated with the vaccines (19).

Dead tumor cells also released DNA fragments into the plasma, similarly to the case of HMGB1. However, plasma cfDNA contains not only tumor-derived DNA fragments due to pathogenic cell death but also normal cell-derived DNA fragments due to physiologic death (20). The physiologic death of normal cells is mainly caused by apoptosis and it generates DNA fragments of $<200$ bp in length. In contrast, pathogenic death of tumor cells is mainly due to necrosis and generates DNA fragments of more random size, including some of longer length (21). To quantify the plasma cfDNA fragments derived from dead cells caused by pathogenic and 
Table I. Cox's proportional hazard analysis of various factors with OS.

\begin{tabular}{lcr}
\hline Factors & Hazard ratio $(95 \%$ CI $)$ & P-value \\
\hline Age $(1$-year increase) & $0.98(0.93-1.05)$ & 0.661 \\
Lymphocyte $(>1,200$ or less $)$ & $0.68(0.26-1.74)$ & 0.418 \\
Pathogenetic classification & $0.29(0.07-1.14)$ & 0.077 \\
Type I vs. type II & & 0.918 \\
Vaccine induced immune response & $0.94(0.31-2.80)$ & 0.539 \\
IgG response & $1.44(0.45-5.50)$ & \\
CTL response & $0.69(0.35-1.05)$ & 0.097 \\
cfDNA integrity $(0.01$ increase) & $0.27(0.04-0.94)$ \\
Pre-vaccination & $1.17(0.77-2.17)$ & 0.038 \\
Post-vaccination & & 0.509 \\
$\Delta$ (Post-Pre) &
\end{tabular}

OS, overall survival; cfDNA, circulating cell-free DNA; CI, confidence interval; CTL, cytotoxic T-lymphocyte.

A
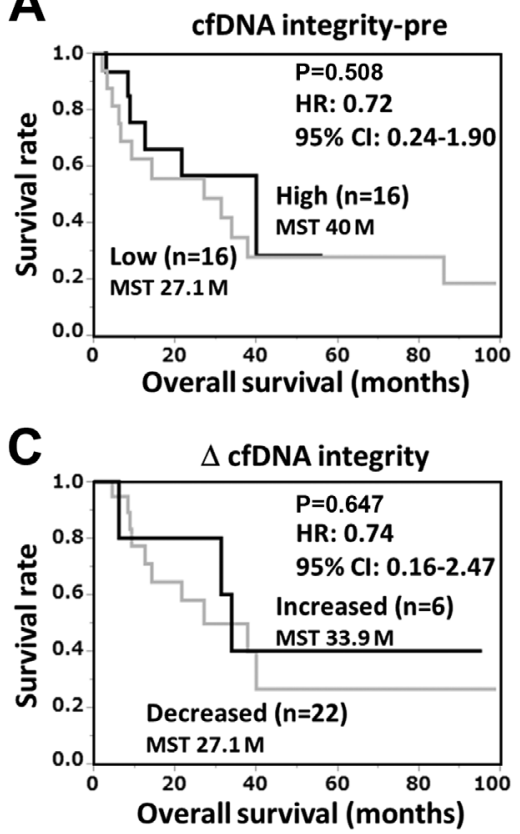

B

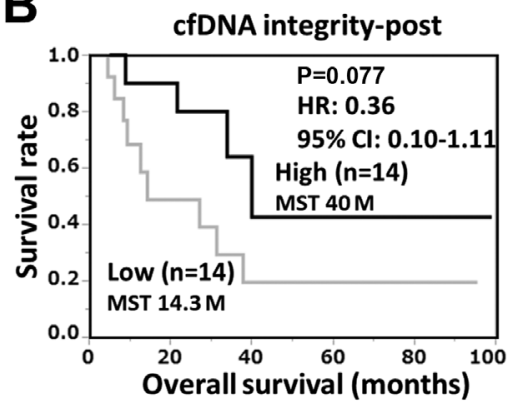

(2) 
by the vaccination converted the necrosis of tumor cells to apoptosis. This possibility was supported by the negative correlation between the cfDNA integrity and vaccine-induced immune responses found in ovarian cancer (9). However, this idea cannot fully explain the correlation between the cfDNA integrity and prognosis. Vaccination may induce not only CTL-mediated apoptosis but also necrosis or other types of cell death by various effector mechanisms, including antibody-dependent cell lysis. In addition, most patients had a history of previous chemotherapy and the carryover of the effect was not neglected. The plasma cfDNA integrity may reflect the comprehensive effects of these therapies.

In conclusion, we investigated the circulating cfDNA integrity of patients with advanced endometrial cancer during treatment with a personalized peptide vaccination and we found that: i) plasma cfDNA integrity was decreased during vaccination, and ii) cfDNA integrity was correlated with prognosis. Some of these findings have been confirmed in other cancers, and thus the cfDNA integrity might be an important marker for future cancer vaccine therapies in general, and might also be applicable for other immune therapies.

\section{Acknowledgements}

The authors would like to thank Ms. M. Ozawa, K. Yamamoto for technical assistance, and K. Yanaga for secretarial assistance. All from the Cancer Vaccine Development Division, Research Center for Innovative Cancer Therapy, Kurume University.

\section{Funding}

The present study was supported by the Kurume University Research Branding Project.

\section{Availability of data and materials}

The datasets used and/or analyzed during the present study are available from the corresponding author on reasonable request.

\section{Authors' contributions}

KW and AY contributed to the study conception and design. Clinical sample collection was performed by KK and NT. Material preparation, data collection and analysis were performed by KW, KY and NK. The first draft of the manuscript was written by KW and AY, and all authors commented on previous versions of the manuscript. All authors read and approved the final manuscript.

\section{Ethics approval and consent to participate}

All procedures performed in studies involving human participants were in accordance with the ethical standards of the institutional and/or national research committee and with the 1964 Helsinki declaration and its later amendments or comparable ethical standards. The clinical studies were approved by the Kurume University Ethics Committee and registered with the UMIN Clinical Trial Registry under trial numbers UMIN1482, 10068, 11230 and 14855.

\section{Patient consent for publication}

Not applicable.

\section{Competing interests}

Akira Yamada is a board member of the Bright Path Biotherapeutics (Kawasaki, Japan).

\section{References}

1. Yamagami W, Nagase $S$, Takahashi F, Ino K, Hachisuga $T$, Aoki D and Katabuchi H: Clinical statistics of gynecologic cancers in Japan. J Gynecol Oncol 28: e32, 2017.

2. Bray F, Ferlay J, Soerjomataram I, Siegel RL, Torre LA and Jemal A: Global cancer statistics 2018: GLOBOCAN estimates of incidence and mortality worldwide for 36 cancers in 185 countries. CA Cancer J Clin 68: 394-424, 2018.

3. McDonald ME and Bender DP: Endometrial cancer: Obesity, genetics, and targeted agents. Obstet Gynecol Clin North Am 46: 89-105, 2019.

4. Yamada A, Sasada T, Noguchi M and Itoh K: Next-generation peptide vaccines for advanced cancer. Cancer Sci 104: 15-21, 2013.

5. Sasada T, Yamada A, Noguchi M and Itoh K: Personalized peptide vaccine for treatment of advanced cancer. Curr Med Chem 21: 2332-2345, 2014.

6. Sakamoto S, Noguchi M, Yamada A, Itoh K and Sasada T: Prospect and progress of personalized peptide vaccinations for advanced cancers. Expert Opin Biol Ther 16: 689-698, 2016.

7. Kawano K, Tsuda N, Matsueda S, Sasada T, Watanabe N, Ushijima K, Yamaguchi T, Yokomine M, Itoh K, Yamada A and Kamura T: Feasibility study of personalized peptide vaccination for recurrent ovarian cancer patients. Immunopharmacol Immunotoxicol 36: 224-236, 2014.

8. Kawano K, Tsuda N, Waki K, Matsueda S, Hata Y, Ushijima K, Itoh K, Yamada A and Kamura T: Personalized peptide vaccination for cervical cancer patients who have received prior platinum-based chemotherapy. Cancer Sci 106: 1111-1117, 2015.

9. Waki K, Yokomizo K, Kawano K, Tsuda N, Komatsu N and Yamada A: Integrity of plasma DNA is inversely correlated with vaccine-induced antitumor immunity in ovarian cancer patients. Cancer Immunol Imunother 69: 2001-2007, 2020.

10. Umetani N, Kim J, Hiramatsu S, Reber HA, Hines OJ, Bilchik AJ and Hoon DS: Increased integrity of free circulating DNA in sera of patients with colorectal or periampullary cancer: Direct quantitative PCR for ALU repeats. Clin Chem 52: 1062-1069, 2006.

11. Livak KJ and Schmittgen TD: Analysis of relative gene expression data using real-time quantitative PCR and the 2(-Delta Delta C(T)) method. Methods 25: 402-408, 2001.

12. Noguchi M, Mine T, Komatsu N, Suekane S, Moriya F, Matsuoka K, Yutani S, Shichijo S, Yamada A, Toh U, et al: Assessment of immunological biomarkers in patients with advanced cancer treated by personalized peptide vaccination. Cancer Biol Ther 10: 1266-1279, 2010.

13. Shirahama T, Muroya D, Matsueda S, Yamada A, Shichijo S, Naito M, Yamashita T, Sakamoto S, Okuda K, Itoh K, et al: A randomized phase II trial of personalized peptide vaccine with low dose cyclophosphamide in biliary tract cancer. Cancer Sci 108: 838-845, 2017.

14. Kibe S, Yutani S, Motoyama S, Nomura T, Tanaka N, Kawahara A, Yamaguchi T, Matsueda S, Komatsu N, Miura M, et al: Phase II study of personalized peptide vaccination for previously treated advanced colorectal cancer. Cancer Immunol Res 2: 1154-1162, 2014.

15. Yutani S, Komatsu N, Yoshitomi M, Matsueda S, Yonemoto K, Mine T, Noguchi M, Ishihara Y, Yamada A, Itoh K and Sasada T: A phase II study of a personalized peptide vaccination for chemotherapy-resistant advanced pancreatic cancer patients. Oncol Rep 30: 1094-1100, 2013. 
16. Yoshitomi M, Yutani S, Matsueda S, Ioji T, Komatsu N, Shichijo S, Yamada A, Itoh K, Sasada T and Kinoshita H: Personalized peptide vaccination for advanced biliary tract cancer: IL-6, nutritional status and pre-existing antigen-specific immunity as possible biomarkers for patient prognosis. Exp Ther Med 3: 463-469, 2012.

17. Yoshiyama K, Terazaki Y, Matsueda S, Shichijo S, Noguchi M, Yamada A, Mine T, Ioji T, Itoh K, Shirouzu K, et al: Personalized peptide vaccination in patients with refractory non-small cell lung cancer. Int J Oncol 40: 1492-1500, 2012.

18. Fucikova J, Moserova I, Urbanova L, Bezu L, Kepp O, Cremer I, Salek C, Strnad P, Kroemer G, Galluzzi L and Spisek R: Prognostic and predictive value of DAMPs and DAMP-associated processes in cancer. Front Immunol 6: 402, 2015

19. Waki K, Kawano K, Tsuda N, Ushijima K, Itoh K and Yamada A: Plasma levels of high-mobility group box 1 during peptide vaccination in patients with recurrent ovarian cancer. J Immunol Res 2017: 1423683, 2017.
20. Jin Z and El-Deiry WS: Overview of cell death signaling pathways. Cancer Biol Ther 4: 139-163, 2005.

21. Giacona MB, Ruben GC, Iczkowski KA, Roos TB, Porter DM and Sorenson GD: Cell-free DNA in human blood plasma: Length measurements in patients with pancreatic cancer and healthy controls. Pancreas 17: 89-97, 1998.

22. Cheng J, Tang Q, Cao X and Burwinkel B: Cell-free circulating DNA integrity based on peripheral blood as a biomarker for diagnosis of cancer: A systematic review. Cancer Epidemiol Biomarkers Prev 26: 1595-1602, 2017.

23. Zhang R, Pu W, Zhang S, Chen L, Zhu W, Xiao L, Xing C and Li K: Clinical value of ALU concentration and integrity index for the early diagnosis of ovarian cancer: A retrospective cohort trial. PLoS One 13: e0191756, 2018.

(c) (1) () This work is licensed under a Creative Commons c. 6 EY No No Attribution-NonCommercial-NoDerivatives 4.0 International (CC BY-NC-ND 4.0) License. 